International Journal of Pure and Applied Mathematics

Volume 103 No. 3 2015, 523-535

ISSN: 1311-8080 (printed version); ISSN: 1314-3395 (on-line version)

url: http://www.ijpam.eu

doi: http://dx.doi.org/10.12732/ijpam.v103i3.13

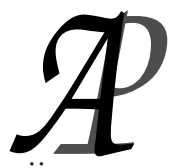

ijpam.eu

\title{
CONTINUED FRACTION FOR TRIBONACCI RATIO
}

\author{
Eunmi Choi \\ Department of Mathematics \\ HanNam University \\ Daejeon, KOREA
}

Abstract: We study generalized continued fractions for the expression of tribonacci ratios $\frac{T_{n+k}}{T_{n}}$ when $n$ is large, and find $n$th convergents of the continued fraction explicitly for each $n$.

AMS Subject Classification: 11B37, 11B39

Key Words: tribonacci sequence, generalized continued fraction

\section{Introduction}

The tribonacci sequence $\left\{T_{n}\right\}$ is a generalized fibonacci sequence defined by $T_{n}=T_{n-1}+T_{n-2}+T_{n-3}$ with $T_{0}=0$ and $T_{1}=T_{2}=1$, so $\left\{T_{n}\right\}=\{0,1,1,3,4,7,13, \cdots\}$. It is well known that the fibonacci ratio $\frac{F_{n+1}}{F_{n}}$ with large $n$ satisfies the fibonacci polynomial $x^{2}=x+1$. Due to continued fraction (CF for short) expansion for quadratic polynomial, the ratio is exhibited by $\langle 1 ; 1,1, \cdots\rangle$. Similar to this, the tribonacci ratio shows

$$
\frac{T_{n+1}}{T_{n}}=\frac{T_{n}+T_{n-1}+T_{n-2}}{T_{n}}=1+\frac{1}{\frac{T_{n}}{T_{n-1}}}+\frac{1}{\frac{T_{n}}{T_{n-1}} \frac{T_{n-1}}{T_{n-2}}},
$$

so when $n$ is large, $\frac{T_{n+1}}{T_{n}}$ holds the tribonacci polynomial $x^{3}=x^{2}+x+1$.

The CF has been used to solve many problems not only in mathematics but

Received: May 13, 2015

(c) 2015 Academic Publications, Ltd. url: www.acadpubl.eu 
in engineering, physics and computer field, since it provides good approximations of roots of quadratic polynomials. However real roots of cubic polynomials may not be naturally explained by means of classical CF. A question of generalization of $\mathrm{CF}$ to higher degree polynomial has been raised by Hermite in 1850 [7]. However, as is mentioned in [6] the CF expression even for cubic polynomial has not been studied extensively. Among few researches, [4] and [5] made use of bifurcating $\mathrm{CF}$ for cubic polynomial. It was called as a ternary CF in [3].

This work is devoted to investigating tribonacci ratios with respect to $\mathrm{CF}$ expressions. We will study generalized CFs for the expression of $\frac{T_{n+k}}{T_{n}}$ when $n$ is large, and also find $n$th convergent of CF explicitly for each $n$.

\section{Continued Fraction for Cubic Polynomial}

We shall denote a finite $\mathrm{CF}$

$$
a_{0}+\frac{b_{1}}{a_{1}+\frac{b_{2}}{\ddots_{++\frac{b_{n}}{a_{n}}}}}=a_{0}+\frac{b_{1}}{a_{1}+\frac{b_{2}}{a_{2}+\cdots+}} \frac{b_{n}}{a_{n}}
$$

by a double CF $\left\langle a_{0} ; \begin{array}{l}b_{1}, b_{2} \\ a_{1}, a_{2}\end{array}, \cdots,{ }_{a_{n}}^{b_{n}}\right\rangle$. If all $b_{i}=1$ then $\left\langle a_{0} ; \underset{a_{1}}{1}, \cdots,{ }_{a_{n}}^{1}\right\rangle$ represent a simple $\mathrm{CF}\left\langle a_{0} ; a_{1}, \cdots, a_{n}\right\rangle$. Moreover an infinite double $\mathrm{CF}$ is defined by

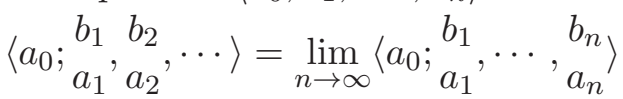

Let $\alpha$ be a real root of $x^{3}=x^{2}+x+1$. Then

$$
\alpha-1=\frac{\alpha^{2}(\alpha-1)}{\alpha^{2}}=\frac{\alpha+1}{\alpha^{2}}=\frac{1}{(\alpha-1)+\frac{1}{\alpha+1}}
$$

shows a simple CF $\alpha=\langle 1 ; \alpha-1, \alpha+1\rangle$. And we also have

$$
\alpha=1+\frac{1}{\frac{\alpha^{2}}{\alpha+1}}=1+\frac{1}{\frac{\alpha(\alpha+1)-\alpha}{\alpha+1}}=1+\frac{1}{\alpha-\frac{1}{1+\frac{1}{\alpha}}}=\langle 1 ; \alpha,-1,-\alpha\rangle .
$$

On the other hand since $\alpha+1=2+\frac{1}{\alpha-\frac{1}{1+\frac{1}{\alpha}}}$, it follows that

$$
\alpha=1+\frac{1}{(\alpha-1)+\frac{1}{\alpha+1}}=1+\frac{1}{\frac{1}{\alpha-\frac{1}{1+\frac{1}{\alpha}}}+\frac{1}{2+\frac{1}{\alpha-\frac{1}{1+\frac{1}{\alpha}}}}} .
$$


So if we let $\frac{1}{\alpha-\frac{1}{1+\frac{1}{\alpha}}}=\beta$ then $\alpha=1+\frac{1}{\beta+\frac{1}{\beta+2}}=\langle 1 ; \beta, \beta+2\rangle$ where $\beta=$ $\frac{1}{\alpha+} \frac{1}{-1+} \frac{1}{-\alpha}=\langle 0 ; \alpha,-1,-\alpha\rangle$.

Besides these, there could be various simple CF expressions for the root of $x^{3}=x^{2}+x+1$. But they do not seem like satisfactory forms to find real root explicitly. We now shall deal with double CF expansion.

Theorem 1. For a real root $\alpha$ of $x^{3}=x^{2}+x+1$, a double $C F$ expansion is $\alpha=\left\langle 1 ; \begin{array}{c}\beta \\ 1\end{array}{ }_{1}^{\prime}, 1, \ldots\right\rangle$ with $\beta=\left\langle 1 ; \begin{array}{c}1 \\ 1\end{array}, 1,1, \ldots\right\rangle$

Proof. The polynomial $x^{3}=x^{2}+x+1$ yields

$$
\alpha=1+\frac{\alpha+1}{\alpha^{2}}=1+\frac{\alpha+1}{\alpha} \frac{1}{\alpha} .
$$

Let $\beta=\frac{\alpha+1}{\alpha}$. Then

$$
\alpha=1+\frac{\beta}{\alpha}=1+\frac{\beta}{1+\frac{\beta}{\alpha}}=1+\frac{\beta}{1+\frac{\beta}{1+\frac{\beta}{\alpha}}}=\cdots=\left\langle 1 ;{ }_{1}{ }^{\prime}{ }_{1}{ }^{\prime}{ }_{1}, \cdots\right\rangle
$$

while $\beta=1+\frac{1}{\alpha}=1+\frac{1}{1+\frac{\beta}{\alpha}}=\left\langle 1 ;{ }_{1}^{1}{ }^{\prime} \alpha^{\beta}\right\rangle=\left\langle 1 ;{ }_{1}{ }^{\prime}{ }_{1}{ }^{\prime}{ }_{1}, \cdots\right\rangle$.

Let $a_{i}, b_{i}(i \geq 0)$ be integers and $\alpha_{i}, \beta_{i}(i \geq 1)$ be nonzero integers. Suppose that a real number $\alpha=\alpha_{0}$ bifurcates into $a_{0}$ and $\frac{\beta_{1}}{\alpha_{1}}$, i.e., $\alpha_{0}=a_{0}+\frac{\beta_{1}}{\alpha_{1}}$. And assume that $\alpha_{i}=a_{i}+\frac{\beta_{i+1}}{\alpha_{i+1}}$ and $\beta_{i}=b_{i}+\frac{1}{\alpha_{i+1}}$ for all $i \geq 1$. Then the first few level of bifurcations of $\alpha$ and $\beta$ are as follows.

$$
\begin{aligned}
& \alpha=a_{0}+\frac{\beta_{1}}{\alpha_{1}}=a_{0}+\frac{b_{1}+\frac{1}{\alpha_{2}}}{a_{1}+\frac{\beta_{2}}{\alpha_{2}}}=a_{0}+\frac{b_{1}+\frac{1}{a_{2}+\frac{\beta_{3}}{\alpha_{3}}}}{a_{1}+\frac{b_{2}+\frac{1}{\alpha_{3}}}{a_{2}+\frac{\beta_{3}}{\alpha_{3}}}}=\cdots \\
& \beta=\beta_{0}=b_{0}+\frac{1}{\alpha_{1}}=b_{0}+\frac{1}{a_{1}+\frac{\beta_{2}}{\alpha_{2}}}=b_{0}+\frac{1}{a_{1}+\frac{b_{2}+\frac{1}{\alpha_{3}}}{a_{2}+\frac{\beta_{3}}{\alpha_{3}}}}=\cdots
\end{aligned}
$$

The pair of objects representing $\alpha$ and $\beta$ is called a bifurcating $\mathrm{CF}$ and denoted by $(\alpha, \beta)=\left[\left\{a_{0}, a_{1}, \cdots\right\},\left\{b_{0}, b_{1}, \cdots\right\}\right]$ in [1]. Hence the real root $\alpha$ of $x^{3}=$ $x^{2}+x+1$ in Theorem 1 is expressed by $(\alpha, \beta)=[\{1,1, \cdots\},\{1,1, \cdots\}]$. 


\section{Continued Fraction for Tribonacci Ratio}

Let $\alpha_{i}=a_{i}+\frac{\beta_{i+1}}{\alpha_{i+1}}$ and $\beta_{i}=b_{i}+\frac{1}{\alpha_{i+1}}$ for every $i>0$. Then due to $(\ddagger)$, the $i$ th bifurcation $\alpha^{[i]}$ of $\alpha=\alpha_{0}$ can be written by

$$
\alpha=\alpha^{[i]}=a_{0}+\frac{N_{i}(i)}{D_{i}(i)}
$$

where $\alpha^{[1]}=a_{0}+\frac{N_{1}(1)}{D_{1}(1)}$ with $N_{1}(1)=\beta_{1}, D_{1}(1)=\alpha_{1}$,

$\alpha^{[2]}=a_{0}+\frac{N_{2}(2)}{D_{2}(2)}$ with $N_{2}(2)=b_{1}+\frac{1}{\alpha_{2}}, \quad D_{2}(2)=a_{1}+\frac{\beta_{2}}{\alpha_{2}}$,

$\alpha^{[3]}=a_{0}+\frac{N_{3}(3)}{D_{3}(3)}$ with $N_{3}(3)=b_{1}+\frac{1}{a_{2}+} \frac{\beta_{3}}{\alpha_{3}}, D_{3}(3)=a_{1}+\frac{b_{2}+\frac{1}{\alpha_{3}}}{a_{2}+} \frac{\beta_{3}}{\alpha_{3}}$,

and so on.

Now for $i \leq j$, let $N_{i}(j)$ denote the same formula of $N_{i}(i)$ where all values $a_{s}, b_{s}, \alpha_{s}$ and $\beta_{s}$ in $N_{i}(i)$ are replaced by $a_{s+(j-i)}, b_{s+(j-i)}, \alpha_{s+(j-i)}$ and $\beta_{s+(j-i)}$ in $N_{i}(j)$. We also define $D_{i}(j)$ analogously from $D_{i}(i)$. For instance $N_{3}(6)=$ $b_{4}+\frac{1}{a_{5}+} \frac{\beta_{6}}{\alpha_{6}}$ and $D_{3}(5)=a_{3}+\frac{b_{4}+\frac{1}{\alpha_{5}}}{a_{4}+} \frac{\beta_{5}}{\alpha_{5}}$.

Theorem 2. With the same context above, the ith bifurcation of $\alpha=\alpha_{0}$ forms $\alpha^{[i]}=a_{0}+\frac{N_{i}(i)}{D_{i}(i)}$, where $N_{i}(i), D_{i}(i)$ are written by double $C F$ that $N_{1}(1)=N_{2}(2)=\left\langle b_{1} ; D_{1}(2)\right\rangle, \quad D_{1}(1)=D_{2}(2)=\left\langle a_{1} ; \begin{array}{c}N_{1}(2) \\ D_{1}(2)\end{array}\right\rangle$

and for all $i>2$,

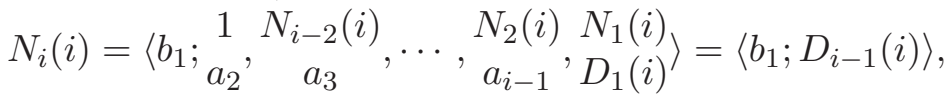

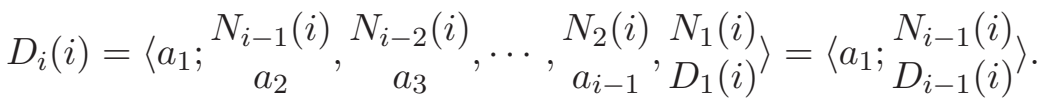

Proof. The $N_{i}(i), D_{i}(i)$ in $\alpha^{[i]}$ follow inductively from ( $\left.\ddagger\right)$ :

(i) $N_{1}(1)=\beta_{1}$ and $D_{1}(1)=\alpha_{1}$.

(ii) $N_{2}(2)=b_{1}+\frac{1}{\alpha_{2}}=b_{1}+\frac{1}{D_{1}(2)}=\left\langle b_{1} ; D_{1}(2)\right\rangle$,

$$
D_{2}(2)=a_{1}+\frac{\beta_{2}}{\alpha_{2}}=\left\langle a_{1} ; \begin{array}{l}
N_{1}(2) \\
D_{1}(2)
\end{array}\right\rangle .
$$

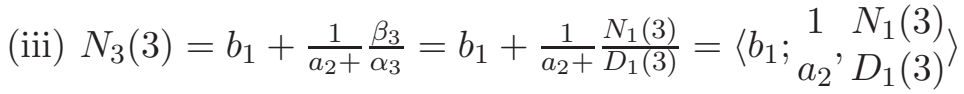

$$
\begin{aligned}
& =b_{1}+\frac{1}{D_{2}(3)}=\left\langle b_{1} ; D_{2}(3)\right\rangle \\
D_{3}(3) & =a_{1}+\frac{b_{2}+\frac{1}{\alpha_{3}}}{a_{2}+} \frac{\beta_{3}}{\alpha_{3}}=a_{1}+\frac{N_{2}(3)}{a_{2}+} \frac{N_{1}(3)}{D_{1}(3)}=\left\langle\begin{array}{cc}
a_{1} ; & N_{2}(3) \\
a_{2} & N_{1}(3) \\
D_{1}(3)
\end{array}\right\rangle \\
& =a_{1}+\frac{N_{2}(3)}{D_{2}(3)}=\left\langle\begin{array}{l}
N_{2}(3) \\
D_{2}(3)
\end{array}\right\rangle .
\end{aligned}
$$


(iv) $N_{4}(4)=b_{1}+\frac{1}{a_{2}+} \frac{b_{3}+\frac{1}{\alpha_{4}}}{a_{3}+} \frac{\beta_{4}}{\alpha_{4}}=b_{1}+\frac{1}{a_{2}+} \frac{N_{2}(4)}{a_{3}+} \frac{N_{1}(4)}{D_{1}(4)}$

$$
\begin{aligned}
& =\left\langle b_{1} ; \begin{array}{c}
1 \\
a_{2},{ }_{2}(4)
\end{array}, \begin{array}{c}
N_{1}(4) \\
D_{1}(4)
\end{array}\right\rangle=b_{1}+\frac{1}{D_{3}(4)}=\left\langle b_{1} ; D_{3}(4)\right\rangle \\
& D_{4}(4)=a_{1}+\frac{b_{2}+\frac{1}{a_{3}+\frac{\beta_{4}}{\alpha_{4}}}}{a_{2}+} \frac{b_{3}+\frac{1}{\alpha_{4}}}{a_{3}+} \frac{\beta_{4}}{\alpha_{4}}=a_{1}+\frac{N_{3}(4)}{a_{2}+} \frac{N_{2}(4)}{a_{3}+} \frac{N_{1}(4)}{D_{1}(4)}
\end{aligned}
$$

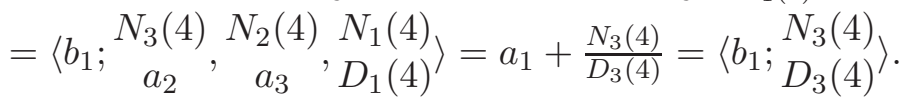

Continuing this process, $N_{i}(i)$ and $D_{i}(i)$ follow that

$b_{1}+\frac{1}{a_{2}+} \frac{N_{i-2}(i)}{a_{3}+} \cdots \frac{N_{2}(i)}{a_{i-1}+} \frac{N_{1}(i)}{D_{1}(i)}=\left\langle b_{1} ; \begin{array}{cc}1 & N_{i-2}(i) \\ a_{2} & a_{3}\end{array}, \cdots, \begin{array}{c}N_{2}(i) \\ a_{i-1}\end{array} \begin{array}{c}N_{1}(i) \\ D_{1}(i)\end{array}\right\rangle$,

$a_{1}+\frac{N_{i-1}(i)}{a_{2}+} \frac{}{a_{3}+} \cdots \frac{N_{2}(i)}{a_{i-1}+} \frac{N_{1}(i)}{D_{1}(i)}=\left\langle a_{1} ; \begin{array}{c}N_{i-1}(i) \\ a_{2}\end{array}, \cdots, \begin{array}{c}N_{2}(i) \\ a_{i-1}, N_{1}(i) \\ D_{1}(i)\end{array}\right\rangle$

respectively.

Moreover we define $\hat{N}_{i}(i)$ and $\hat{D}_{i}(i)$ by the same $N_{i}(i)$ and $D_{i}(i)$ respectively, in which the last fractions $\frac{\beta_{i}}{\alpha_{i}}$ and $\frac{1}{\alpha_{i}}$ are omitted.

Theorem 3. $\hat{N}_{i}(i)$ and $\hat{D}_{i}(i)$ satisfy the followings.

(1) For $i=1,2, \hat{N}_{i}(i)=b_{1}$ and $\hat{D}_{i}(i)=a_{1}$.

(2) For $i>2, \hat{N}_{i}(i)=\left\langle b_{1} ; \hat{D}_{i-1}(i)\right\rangle$ and $\hat{D}_{i}(i)=\left\langle a_{1} ; \begin{array}{l}\hat{N}_{i-1}(i) \\ \hat{D}_{i-1}(i)\end{array}\right\rangle$. In particular $\hat{N}_{i}(i)=\left\langle 1 ; \hat{D}_{i-1}(i-1)\right\rangle$ and $\hat{D}_{i}(i)=\left\langle 1 ; \begin{array}{c}\hat{N}_{i-1}(i-1) \\ \hat{D}_{i-1}(i-1)\end{array}\right\rangle$, if $a_{i}=b_{i}=1$ for all $i$.

(3) $\lim _{i \rightarrow \infty} \frac{\hat{N}_{i}(i)}{\hat{D}_{i}(i)}=\lim _{i \rightarrow \infty} \frac{N_{i}(i)}{D_{i}(i)}$.

Proof. It is clear that $\hat{N}_{1}(1)=\hat{N}_{2}(2)=b_{1}$ and $\hat{D}_{1}(1)=\hat{D}_{2}(2)=a_{1}$. And

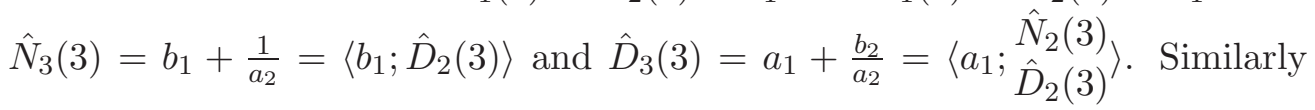
$\hat{N}_{4}(4)=b_{1}+\frac{1}{a_{2}+\frac{b_{3}}{a_{3}}}=\left\langle b_{1} ; \hat{D}_{3}(4)\right\rangle$ and $\hat{D}_{4}(4)=a_{1}+\frac{b_{2}+\frac{1}{a_{3}}}{a_{2}+\frac{b_{3}}{a_{3}}}=\left\langle\begin{array}{l}\hat{N}_{1} ; \\ \hat{N}_{3}(4)\end{array}\right\rangle$. So we have $\hat{N}_{i}(i)=\left\langle b_{1} ; \hat{D}_{i-1}(i)\right\rangle$ and $\hat{D}_{i}(i)=\left\langle a_{1} ; \begin{array}{c}\hat{N}_{i-1}(i) \\ \hat{D}_{i-1}(i)\end{array}\right\rangle$ for all $i>2$.

Obviously $\hat{N}_{2}(2)$ and $\hat{D}_{2}(2)$ are the 1st convergents of $N_{2}(2)$ and $D_{2}(2)$ respectively. Moreover $\hat{N}_{3}(3)=\left\langle b_{1} ; \begin{array}{c}1 \\ a_{2}\end{array}\right\rangle$ and $\hat{D}_{3}(3)=\left\langle a_{1} ; \begin{array}{c}b_{2} \\ a_{2}\end{array}\right\rangle=\left\langle a_{1} ; \begin{array}{c}\hat{N}_{2}(3) \\ a_{2}\end{array}\right\rangle$ are the 2nd convergents of $N_{3}(3)=\left\langle b_{1} ; \begin{array}{c}1 \\ a_{2}, \alpha_{3}\end{array}\right\rangle$ and $D_{3}(3)=\left\langle a_{1} ; \begin{array}{c}N_{2}(3) \\ a_{2}\end{array} \begin{array}{c}N_{1}(3) \\ D_{1}(3)\end{array}\right\rangle$ respectively. Continuing this consideration we may say $\hat{N}_{i}(i)$ and $\hat{D}_{i}(i)$ are 
the $(i-1)$ th convergents of $N_{i}(i)$ and $D_{i}(i)$ respectively. Hence it follows that $\lim _{i \rightarrow \infty} \frac{\hat{N}_{i}(i)}{\hat{D}_{i}(i)}=\lim _{i \rightarrow \infty} \frac{N_{i}(i)}{D_{i}(i)}$.

In particular if $a_{i}=b_{i}=1$ then the bifurcation

$$
\alpha=\alpha_{0}=1+\frac{\beta_{1}}{\alpha_{1}}=1+\frac{1+\frac{1}{\alpha_{2}}}{1+\frac{\beta_{2}}{\alpha_{2}}}=1+\frac{1+\frac{1}{1+\frac{\beta_{3}}{\alpha_{3}}}}{1+\frac{1+\frac{1}{\alpha_{3}}}{1+\frac{\beta_{3}}{\alpha_{3}}}}=\cdots
$$

implies that

$$
\begin{aligned}
& \hat{N}_{2}(2)=\hat{D}_{2}(2)=1 \text { and } \hat{N}_{3}(3)=\hat{D}_{3}(3)=1+\frac{1}{1}=2, \\
& \hat{N}_{4}(4)=1+\frac{1}{1+\frac{1}{1}}=1+\frac{1}{\hat{D}_{3}(3)}, \hat{D}_{4}(4)=1+\frac{1+\frac{1}{1}}{1+\frac{1}{1}}=1+\frac{\hat{N}_{3}(3)}{\hat{D}_{3}(3)},
\end{aligned}
$$

and so on. Therefore we have

$$
\begin{aligned}
& \hat{N}_{i}(i)=1+\frac{1}{\hat{D}_{i-1}(i-1)}=\left\langle 1 ; \hat{D}_{i-1}(i-1)\right\rangle \\
& \hat{D}_{i}(i)=1+\frac{\hat{N}_{i-1}(i-1)}{\hat{D}_{i-1}(i-1)}=\left\langle 1 ; \begin{array}{c}
\hat{N}_{i-1}(i-1) \\
\hat{D}_{i-1}(i-1)
\end{array}\right\rangle \text {. }
\end{aligned}
$$

Theorem 4. Let $\alpha=\lim _{n \rightarrow \infty} \frac{T_{n+1}}{T_{n}}$. Then we have the followings.

(1) The double $C F \alpha$ is $\alpha=\left\langle 1 ; \begin{array}{c}\beta \\ 1\end{array}, \begin{array}{l}\beta \\ 1\end{array}, \cdots\right\rangle$ with $\beta=\left\langle 1 ; \begin{array}{c}1 \\ 1\end{array}, \begin{array}{l}\beta \\ 1\end{array}, \cdots\right\rangle$.

(2) The ith bifurcation is $\alpha^{[i]}=1+\frac{N_{i}(i)}{D_{i}(i)}$, where $N_{1}(1)=\beta, D_{1}(1)=\alpha$ and $N_{i}(i)=1+\frac{1}{D_{i-1}(i-1)}, D_{i}(i)=1+\frac{N_{i-1}(i-1)}{\hat{D}_{i-1}(i-1)}$ for all $i>1$.

(3) $\lim _{i \rightarrow \infty} \frac{N_{i}(i)}{D_{i}(i)}=\lim _{i \rightarrow \infty} \frac{\hat{N}_{i}(i)}{\hat{D}_{i}(i)}$, and $\lim _{n \rightarrow \infty} \frac{T_{n+1}}{T_{n}}=1.8392 \cdots$.

Proof. When $n$ is large, $\alpha=\frac{T_{n+1}}{T_{n}}$ satisfies $x^{3}=x^{2}+x+1$, so (1) is due to Theorem 1. Moreover by letting $\beta=\frac{\alpha+1}{\alpha}$,

$$
\alpha=1+\frac{\beta}{\alpha}=1+\frac{\beta}{1+\frac{\beta}{\alpha}}=1+\frac{\beta}{1+\frac{\beta}{1+\frac{\beta}{\alpha}}}=\cdots
$$

implies $\alpha$ bifurcates into 1 and $\frac{\beta}{\alpha}$, while $\beta$ into 1 and $\frac{1}{\alpha}$. Thus

$$
\alpha=1+\frac{\beta}{\alpha}=1+\frac{1+\frac{1}{\alpha}}{1+\frac{\beta}{\alpha}}=1+\frac{1+\frac{1}{1+\frac{\beta}{\alpha}}}{1+\frac{1+\frac{1}{\alpha}}{1+\frac{\beta}{\alpha}}}=\cdots
$$

Now owing to Theorem 2 and 3, we have $\alpha^{[i]}=1+\frac{N_{i}(i)}{D_{i}(i)}$ where $N_{1}(1)=\beta, D_{1}(1)=\alpha$ 


$$
\begin{aligned}
& N_{2}(2)=1+\frac{1}{\alpha}=1+\frac{1}{D_{1}(1)}, D_{2}(2)=1+\frac{\beta}{\alpha}=1+\frac{N_{1}(1)}{D_{1}(1)} \\
& N_{3}(3)=1+\frac{1}{1+\frac{\beta}{\alpha}}=1+\frac{1}{D_{2}(2)}, D_{3}(3)=1+\frac{1+\frac{1}{\alpha}}{1+\frac{\beta}{\alpha}}=1+\frac{N_{2}(2)}{D_{2}(2)} \\
& N_{4}(4)=1+\frac{1}{1+\frac{1+\frac{1}{\alpha}}{1+\frac{\beta}{\alpha}}}=1+\frac{1}{D_{3}(3)}, D_{4}(4)=1+\frac{N_{3}(3)}{D_{3}(3)}, \cdots
\end{aligned}
$$

thus $N_{i}(i)=1+\frac{1}{D_{i-1}(i-1)}, D_{i}(i)=1+\frac{N_{i-1}(i-1)}{D_{i-1}(i-1)}$ for all $i>1$. Moreover for $\hat{N}_{i}(i)$ and $\hat{D}_{i}(i)$, we have $\hat{N}_{2}(2)=1, \hat{D}_{2}(2)=1$, and $\hat{N}_{3}(3)=1+\frac{1}{1}=1+\frac{1}{\hat{D}_{2}(2)}, \hat{D}_{3}(3)=1+\frac{1}{1}=1+\frac{\hat{N}_{2}(2)}{\hat{D}_{2}(2)}$, $\hat{N}_{4}(4)=1+\frac{1}{1+\frac{1}{1}}=1+\frac{1}{\hat{D}_{3}(3)}, \hat{D}_{4}(4)=1+\frac{1}{1+\frac{1}{1}}=1+\frac{\hat{N}_{3}(3)}{\hat{D}_{3}(3)}$, so $\hat{N}_{i}(i)=1+\frac{1}{\hat{D}_{i-1}(i-1)}$ and $\hat{D}_{i}(i)=1+\frac{\hat{N}_{i-1}(i-1)}{\hat{D}_{i-1}(i-1)}$ for all $i>2$. So by computing the first some $\hat{N}_{i}(i)$ and $\hat{D}_{i}(i)$ explicitly, we have

\begin{tabular}{c|cccccccccc}
$i$ & 2 & 3 & 4 & 5 & 6 & 7 & 8 & 9 & 10 & 11 \\
\hline$\hat{N}_{i}(i)$ & 1 & 2 & $\frac{3}{2}$ & $\frac{3}{2}$ & $\frac{11}{7}$ & $\frac{20}{13}$ & $\frac{37}{24}$ & $\frac{68}{44}$ & $\frac{125}{81}$ & $\frac{230}{149}$ \\
$\hat{D}_{i}(i)$ & 1 & 2 & 2 & $\frac{7}{4}$ & $\frac{13}{7}$ & $\frac{24}{13}$ & $\frac{44}{24}$ & $\frac{81}{44}$ & $\frac{149}{81}$ & $\frac{274}{149}$
\end{tabular}

So the table shows $\frac{\hat{N}_{i}(i)}{\hat{D}_{i}(i)}(2 \leq i \leq 13)$ approximates to $0.8392 \cdots$.

\begin{tabular}{l|l||l|l||l|l}
$i$ & $\hat{N}_{i}(i) / \hat{D}_{i}(i)$ & $i$ & $\hat{N}_{i}(i) / \hat{D}_{i}(i)$ & $i$ & $\hat{N}_{i}(i) / \hat{D}_{i}(i)$ \\
\hline 2 & 1 & 3 & $\frac{2}{2}=1$ & 4 & $\frac{3}{4}=0.75$ \\
5 & $\frac{6}{7}=0.85714$ & 6 & $\frac{11}{13}=0.84615$ & 7 & $\frac{20}{24}=0.83333$ \\
8 & $\frac{37}{44}=0.84090$ & 9 & $\frac{68}{81}=0.83950$ & 10 & $\frac{125}{149}=0.83892$ \\
11 & $\frac{230}{274}=0.83941$ & 12 & $\frac{423}{504}=0.83928$ & 13 & $\frac{778}{927}=0.83926$ \\
\hline
\end{tabular}

Hence we have $\lim _{n \rightarrow \infty} \frac{T_{n+1}}{T_{n}}=\alpha=\lim _{i \rightarrow \infty} \alpha^{[i]}=1+\lim _{i \rightarrow \infty} \frac{\hat{N}_{i}(i)}{\hat{D}_{i}(i)}=1.8392 \cdots$ by Theorem 3.

Compare it to the real root $\frac{1}{3}(1+\sqrt[3]{19+3 \sqrt{33}}+\sqrt[3]{19-3 \sqrt{33}})$ of $x^{3}=$ $x^{2}+x+1$ which approximates to $1.8392 \cdots$.

Corollary 5. Let $\frac{\hat{N}_{n}(n)}{\hat{D}_{n}(n)}=\frac{p_{n}}{q_{n}}$ with $p_{n}, q_{n} \in \mathbb{N}$. Then $\left\{q_{n}\right\}$ is the (original) tribonacci sequence having initials $1,2,4$, while $\left\{p_{n}\right\}$ is a tribonacci type sequence having initials 1, 2, 3. In particular, $1+\frac{\hat{N}_{n}(n)}{\hat{D}_{n}(n)}=\frac{T_{n+1}}{T_{n}}$ for every $n$.

Proof. It is easy to see that $\left\{p_{n}+q_{n}\right\}$ is a tribonacci sequence with initials $2,4,7$. Thus $\left\{p_{n}+q_{n}\right\}=\left\{T_{n+1}\right\}$ and $1+\frac{\hat{N}_{n}(n)}{\hat{D}_{n}(n)}=1+\frac{p_{n}}{q_{n}}=\frac{T_{n+1}}{T_{n}}$. 
For instance $1+\frac{\hat{N}_{16}(16)}{\hat{D}_{16}(16)}=1+\frac{4841}{5768}=\frac{10609}{5768}=\frac{T_{17}}{T_{16}}$.

\section{Continued Fraction for $k$ Step Tribonacci Ratio}

According to [8], ratios that appear in phyllotaxis are fractions of fibonacci numbers spaced 2 steps such as $8 / 3,13 / 5,21 / 8, \cdots$ rather than consecutive terms. The 2 step fibonacci ratio is expressed by $\mathrm{CF}$ that $\frac{F_{n+2}}{F_{n}}=1+\frac{F_{n+1}}{F_{n}}=$ $1+\langle 1 ; \underbrace{1, \cdots, 1}_{n-1}\rangle=\langle 2 ; \underbrace{1, \cdots, 1}_{n-1}\rangle$. However when $n$ is large, any $k$ step fibonacci

ratio $\frac{F_{n+k}}{F_{n}}=\frac{F_{n+k}}{F_{n+k-1}} \cdots \frac{F_{n+1}}{F_{n}}=\langle 1 ; 1, \cdots\rangle^{k}$ may not be simply represented by CF since arithmetic algorithms of CFs are not trivial. In this section we shall study $k$ step tribonacci ratios $\frac{T_{n+k}}{T_{n}}$ with CF expansions. Let us begin with $k=4$.

Theorem 6. For 4 step tribonacci ratio $\frac{T_{n+4}}{T_{n}}, \lim _{n \rightarrow \infty} \frac{T_{n+4}}{T_{n}}=\left\langle 11 ; \begin{array}{c}\beta \\ 11\end{array}, 11, \cdots\right\rangle$ with $\beta=\left\langle 5 ; \begin{array}{cc}1 & \beta \\ 11 & 11\end{array}, \cdots\right\rangle$.

Proof. $T_{n}$ satisfies the 4 step recurrence rule $T_{n+4}=11 T_{n}+5 T_{n-4}+T_{n-8}$ (see [2]), hence we have

$$
\frac{T_{n+4}}{T_{n}}=11+\frac{5}{\frac{T_{n}}{T_{n-4}}}+\frac{1}{\frac{T_{n}}{T_{n-4}} \frac{T_{n-4}}{T_{n-8}}} .
$$

Then $\alpha=\lim _{n \rightarrow \infty} \frac{T_{n+4}}{T_{n}}$ is a root of $x^{3}=11 x^{2}+5 x+1$. So we have $\alpha=11+$ $\frac{5 \alpha+1}{\alpha} \frac{1}{\alpha}=11+\frac{\beta}{\alpha}$ by letting $\beta=\frac{5 \alpha+1}{\alpha}$. Hence $\alpha$ bifurcates into 11 and $\frac{\beta}{\alpha}$ that

$$
\alpha=11+\frac{\beta}{11+\frac{\beta}{\alpha}}=11+\frac{\beta}{11+\frac{\beta}{11+\frac{\beta}{\alpha}}}=\cdots=\left\langle 11 ; \begin{array}{c}
\beta \\
11
\end{array}{ }_{11}, \cdots\right\rangle
$$

while $\beta$ bifurcates into 5 and $\frac{1}{\alpha}$ that

$$
\beta=5+\frac{1}{11+\frac{y}{\alpha}}=5+\frac{1}{11+\frac{\beta}{11+\frac{\beta}{\alpha}}}=\cdots=\left\langle 5 ; \begin{array}{c}
1 \\
11
\end{array}, \begin{array}{c}
\beta \\
11
\end{array}, \cdots\right\rangle .
$$

We now shall compute $\frac{T_{n+4}}{T_{n}}$ explicitly. 
Theorem 7. Let $n=4 t+r(t, r \in \mathbb{N}, 1 \leq r \leq 4)$ and $\alpha_{t}=\frac{T_{n+4}}{T_{n}}$ be the 4 step tribonacci ratio. Then we have the followings.

(1) The double CF expansion of $\alpha_{t}$ is $\alpha_{t}=\left\langle 11 ; \begin{array}{c}\beta_{t-1}, \ldots, \beta_{2} \beta_{1} \\ 11\end{array},_{11} \alpha_{1}\right.$ for $t \geq 2$, where $\beta_{i}=\left\langle 5 ; \begin{array}{c}1, \beta_{i-2}, \ldots,{ }_{11} \beta_{2} \beta_{1} \\ \beta_{1}\end{array}\right\rangle$ for $3 \leq i<t$. In particular $\alpha_{0}=\frac{T_{4+r}}{T_{r}}, \alpha_{1}=\frac{T_{8+r}}{T_{4+r}}$ and $\beta_{i}=\left\langle 5 ; \alpha_{i-1}\right\rangle$ if $i=1,2$.

(2) The ith bifurcation of $\alpha_{t}$ is $\alpha_{t}{ }^{[i]}=11+\frac{N_{i}(i)}{D_{i}(i)}$, where double CFs of $N_{i}(i)$ and $D_{i}(i)$ are $N_{1}(1)=\beta_{t-1}$ and $D_{1}(1)=\alpha_{t-1}$ and

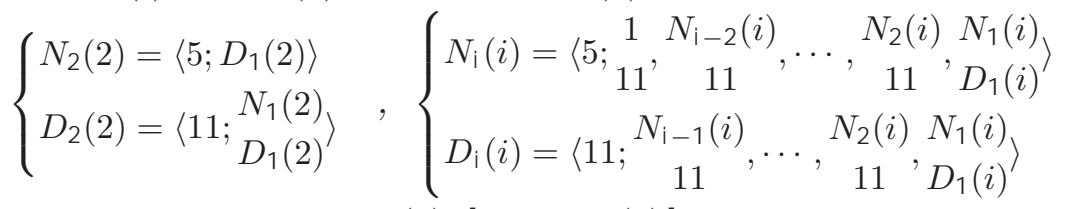

for $3 \leq i<t$. Here $N_{i}(j)$ [resp. $\left.D_{i}(j)\right]$ for $i \leq j$ is defined from $N_{i}(i)$ [resp. $\left.D_{i}(i)\right]$ where $\alpha_{t-i}$ and $\beta_{t-i}$ in $N_{i}(i)$ are replaced by $\alpha_{t-j}$ and $\beta_{t-j}$ in $N_{i}(j)$.

(3) Moreover $\lim _{n \rightarrow \infty} \frac{T_{n+4}}{T_{n}}=11.44452 \cdots$.

Proof. The $T_{4(t+1)+r}=11 T_{4 t+r}+5 T_{4(t-1)+r}+T_{4(t-2)+r}$ shows

$$
\begin{aligned}
\alpha_{t} & =\frac{T_{4(t+1)+r}}{T_{4 t+r}}=11+\frac{5}{\frac{T_{4 t+r}}{T_{4(t-1)+r}}}+\frac{1}{\frac{T_{4 t+r}}{T_{4(t-1)+r}} \frac{T_{4(t-1)+r}}{T_{4(t-2)+r}}} \\
& =11+\frac{5}{\alpha_{t-1}}+\frac{1}{\alpha_{t-1} \alpha_{t-2}}=11+\left(5+\frac{1}{\alpha_{t-2}}\right) \frac{1}{\alpha_{t-1}},
\end{aligned}
$$

so we have $\alpha_{t}=11+\frac{\beta_{t-1}}{\alpha_{t-1}}$ with $\beta_{t-1}=5+\frac{1}{\alpha_{t-2}}$. Thus

$$
\alpha_{t}=11+\frac{\beta_{t-1}}{11+\frac{\beta_{t-2}}{11+\frac{\beta_{t-3}}{\alpha_{t-3}}}}=\cdots=\left\langle 11 ; \begin{array}{c}
\beta_{t-1}, \beta_{t-2}, \ldots, \beta_{2} \beta_{1} \\
11,{ }_{11}{ }^{\prime} \alpha_{1}
\end{array}\right\rangle
$$

for $t \geq 2$, while for $3 \leq i \leq t-1$,

$$
\beta_{i}=5+\frac{1}{11+\frac{\beta_{i-2}}{11+\frac{\beta_{i-3}}{\alpha_{i-3}}}}=\cdots=\left\langle 5 ; \begin{array}{c}
1 \\
11, \beta_{i-2}, \ldots, \beta_{2} \beta_{1} \\
11
\end{array}{ }^{\prime} \alpha_{1}\right.
$$

In particular $\alpha_{0}=\frac{T_{4+r}}{T_{r}}, \alpha_{1}=\frac{T_{4 \cdot 2+r}}{T_{4+r}}$ and $\beta_{2}=5+\frac{1}{\alpha_{1}}=\left\langle 5 ; \alpha_{1}\right\rangle, \beta_{1}=5+\frac{1}{\alpha_{0}}=$ 
$\left\langle 5 ; \alpha_{0}\right\rangle$. On the other hand the bifurcating

$$
\alpha_{t}-11=\frac{\beta_{t-1}}{\alpha_{t-1}}=\frac{5+\frac{1}{\alpha_{t-2}}}{11+\frac{\beta_{t-2}}{\alpha_{t-2}}}=\frac{5+\frac{1}{11+\frac{\beta_{t-3}}{\alpha_{t-3}}}}{11+\frac{5+\frac{1}{\alpha_{t-3}}}{11+\frac{\beta_{t-3}}{\alpha_{t-3}}}}=\cdots
$$

shows the $i$ th level bifurcation $\alpha_{t}{ }^{[i]}=11+\frac{N_{i}(i)}{D_{i}(i)}$ as follows.

$N_{1}(1)=\beta_{t-1}, D_{1}(1)=\alpha_{t-1}$

$N_{2}(2)=\left\langle 5 ; \alpha_{t-2}\right\rangle=\left\langle 5 ; D_{1}(2)\right\rangle, \quad D_{2}(2)=\left\langle 11 ; \begin{array}{l}\beta_{t-2} \\ \alpha_{t-2}\end{array}\right\rangle=\left\langle 11 ; \begin{array}{l}N_{1}(2) \\ D_{1}(2)\end{array}\right\rangle$

$N_{3}(3)=\left\langle 5 ; \begin{array}{cc}1 & \beta_{t-3} \\ 11 & \alpha_{t-3}\end{array}\right\rangle=\left\langle 5 ; \begin{array}{cc}1 & N_{1}(3) \\ 11 & D_{1}(3)\end{array}\right\rangle, D_{3}(3)=\left\langle 11 ; \begin{array}{c}N_{2}(3) \\ 11\end{array} \begin{array}{c}N_{1}(3) \\ D_{1}(3)\end{array}\right\rangle$

$N_{4}(4)=\left\langle 5 ; \begin{array}{ccc}1 & N_{2}(4) & N_{1}(4) \\ 11 & 11 & D_{1}(4)\end{array}\right\rangle, \quad D_{4}(4)=\left\langle 11 ; \begin{array}{cc}N_{3}(4) & N_{2}(4) \\ 11 & N_{1}(4) \\ 11 & D_{1}(4)\end{array}\right\rangle$

and so on, where for $i \leq j$, the $N_{i}(j)$ and $D_{i}(j)$ come from $N_{i}(i)$ and $D_{i}(i)$ in which $\alpha_{t-i}, \beta_{t-i}$ are substituted by $\alpha_{t-j}, \beta_{t-j}$. Thus for all $3 \leq i<t$, we have

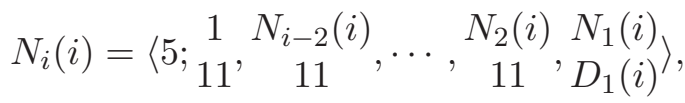

$$
\begin{aligned}
& D_{i}(i)=\left\langle 11 ; \begin{array}{c}
N_{i-1}(i) \\
11
\end{array}, \cdots, \begin{array}{c}
N_{2}(i) \\
11
\end{array}, \begin{array}{l}
N_{1}(i) \\
D_{1}(i)
\end{array}\right\rangle .
\end{aligned}
$$

Furthermore by considering $\hat{N}_{i}(i)$ and $\hat{D}_{i}(i)$ that are the $(i-1)$ th convergent of $N_{i}(i)$ and $D_{i}(i)$ respectively, we have

$\hat{N}_{2}(2)=5, \hat{D}_{2}(2)=11$, so $\frac{\hat{N}_{2}(2)}{\hat{D}_{2}(2)}=\frac{5}{11} \approx 0.454545$,

$\hat{N}_{3}(3)=5+\frac{1}{11}=\frac{56}{11}, \hat{D}_{3}(3)=11+\frac{5}{11}=\frac{126}{11}$, so $\frac{\hat{N}_{3}(3)}{\hat{D}_{3}(3)}=\frac{56}{126} \approx 0.444444$,

$\hat{N}_{4}(4)=\frac{641}{126}, \hat{D}_{4}(4)=\frac{1442}{126}$, so $\frac{\hat{N}_{4}(4)}{\hat{D}_{4}(4)}=\frac{641}{1442} \approx 0.444521$,

and so on. Therefore it follows that

$\lim _{n \rightarrow \infty} \frac{T_{n+4}}{T_{n}}=\lim _{t \rightarrow \infty} \alpha_{t}=11+\lim _{t \rightarrow \infty} \frac{\hat{N}_{t-1}(t-1)}{\hat{D}_{t-1}(t-1)}=11.44452 \cdots$.

Recall the real root $\frac{1}{3}(11+2 \sqrt[3]{199+3 \sqrt{33}}+2 \sqrt[3]{199-3 \sqrt{33}})$ of $x^{3}=11 x^{2}+$ $5 x+1$ that approximates to $11.44452 \cdots$. And it equals $(1.8392 \cdots)^{4}$.

Example. For $\frac{T_{n+4}}{T_{n}}, \hat{N}_{1}(1)=\hat{N}_{2}(2)=5$ and $\hat{D}_{1}(1)=\hat{D}_{2}(2)=11$. And $\hat{N}_{i}(i)=5+\frac{1}{\hat{D}_{i-1}(i-1)}, \hat{D}_{i}(i)=11+\frac{\hat{N}_{i-1}(i-1)}{\hat{D}_{i-1}(i-1)}$ yield the table: 


\begin{tabular}{c|rrrrrr}
$i$ & 2 & \multicolumn{1}{c}{3} & \multicolumn{1}{c}{4} & \multicolumn{1}{c}{5} & \multicolumn{1}{c}{6} & \multicolumn{1}{l}{7} \\
\hline$\hat{N}_{i}(i)$ & 5 & $\frac{56}{11}$ & $\frac{641}{126}$ & $\frac{7336}{1442}$ & $\frac{83957}{16503}$ & $\frac{960848}{188869}$ \\
$\hat{D}_{i}(i)$ & 11 & $\frac{126}{11}$ & $\frac{1442}{126}$ & $\frac{16503}{1442}$ & $\frac{188869}{16503}$ & $\frac{2161516}{188869}$ \\
\hline$\frac{\hat{N}_{i}(i)}{\hat{D}_{i}(i)}$ & & $\frac{56}{126}$ & $\frac{641}{1442}$ & $\frac{7336}{16503}$ & $\frac{83957}{188869}$ & $\frac{960848}{2161516}$ \\
$\approx$ & & .444 & .44452 & .44452 & .44452 & .44452
\end{tabular}

Hence, for instance, the ratio $\frac{T_{38}}{T_{34}}=\frac{T_{4(9)+2}}{T_{4(8)+2}}=\alpha_{8}$ is obtained by the 7 th bifurcation $\alpha_{8}^{[7]}$ that $\alpha_{8}=\alpha_{8}^{[7]}=11+\frac{\hat{N}_{7}(7)}{\hat{D}_{7}(7)} \approx 11.44452 \cdots$.

Moreover all $4 t$ subscripted tribonacci numbers can be retrieved in this way.

Corollary 8. For any $t>1,11+\frac{\hat{N}_{t}(t)}{\hat{D}_{t}(t)}$ retrieves $\frac{T_{4(t+1)}}{T_{4 t}}$.

Proof. Write $11+\frac{\hat{N}_{t}(t)}{\hat{D}_{t}(t)}=\frac{p_{t}}{q_{t}}$. Then the above table shows

\begin{tabular}{c||c|c|c|c|c|c}
$t$ & 2 & 3 & 4 & 5 & 6 & 7 \\
\hline$\frac{p_{t}}{q_{t}}$ & $\frac{126}{11}$ & $\frac{1442}{126}$ & $\frac{16503}{1442}$ & $\frac{188869}{16503}$ & $\frac{2161516}{188869}$ & $\frac{24737524}{2161516}$
\end{tabular}

So we have the set of fractions $\frac{4 p_{t}}{4 q_{t}}$ for $2 \leq t \leq 7$ that

$\left\{\frac{504}{44}, \frac{5768}{504}, \frac{66012}{5768}, \frac{755476}{66012}, \frac{8646064}{755476}, \frac{98950096}{8646064}\right\}$

which equals $\left\{\frac{T_{4(t+1)}}{T_{4 t}}\right\}$. Moreover since $p_{t}=q_{t+1}$ and the set $\left\{q_{t}\right\}=\{11,126,1442$, $16503,188869,2161516\}$ satisfy the recurrence $q_{t+1}=11 q_{t}+5 q_{t-1}+q_{t-2}$, it follows that $\frac{4 p_{t}}{4 q_{t}}=\frac{T_{4(t+1)}}{T_{4 t}}$ for all $t>2$.

Corollary 9. If $n$ is divisible by 4 then so is $T_{n}$.

Proof. $T_{4}=4, T_{8}=44$ and $T_{12}=504$ are all zeros by $\bmod 4$. So if $T_{4 i} \equiv$ $0(\bmod 4)$ for $1 \leq i \leq t$ then $T_{4(t+1)}=11 T_{4 t}+5 T_{4(t-1)}+T_{4(t-2)} \equiv 0(\bmod 4)$.

It is now obvious by Theorem 4 and 7 that any $k$ step tribonacci ratio $\frac{T_{n+k}}{T_{n}}$ depends on the $k$ step recurrence rule for $T_{n}$.

Lemma 1. [2] Any $k$ step recurrence satisfies $T_{n+k}=a_{k} T_{n}+b_{k} T_{n-k}+$ $T_{n-2 k}$ with $a_{k}=3 T_{k}-T_{k-6}$ and $b_{k}=-a_{-k}$. The $\left\{a_{k}\right\}$ and $\left\{b_{k}\right\}$ satisfy $a_{k+3}=a_{k+2}+a_{k+1}+a_{k}$ and $b_{k}=b_{k+1}+b_{k+2}+b_{k+3}$ with initial 1,3,7 and $1,1,-5$, respectively.

In particular the pairs $\left(a_{k}, b_{k}\right)$ for $1 \leq k \leq 10$ are $(1,1),(3,1),(7,-5)$, $(11,5),(21,1),(39,-11),(71,15),(131,-3),(241,-23)$ and $(443,41)$. So for example, $T_{n+3}=7 T_{n}-5 T_{n-3}+T_{n-6}$ and $T_{n+10}=443 T_{n}+41 T_{n-10}+T_{n-20}$. And the next theorem follows immediately. 
Theorem 10. Let $a_{k}, b_{k}$ be integers satisfying $T_{n+k}=a_{k} T_{n}+b_{k} T_{n-k}+$ $T_{n-2 k}$. When $n$ is large, we have the followings.

(1) The $k$ step tribonacci ratio $\frac{T_{n+k}}{T_{n}}$ holds $x^{3}=a_{k} x^{2}+b_{k} x+1$.

(2) If $n=k t+r(1 \leq r \leq k)$ then $\frac{T_{n+k}}{T_{n}}=\left\langle a_{k} ;{ }^{\beta_{t-1}}, \cdots,{ }_{a_{k}},{ }_{a_{k}}{ }^{\prime}{ }^{\beta_{1}}{ }_{\alpha_{1}}\right\rangle$ for $t \geq 2$, with $\beta_{i}=\left\langle b_{k} ; \begin{array}{c}1 \\ a_{k}\end{array}, \begin{array}{c}\beta_{i-2} \\ a_{k}\end{array}, \cdots, \begin{array}{c}\beta_{2} \\ a_{k},{ }^{\prime} \alpha_{1}\end{array}\right\rangle$ for $3 \leq i<t$. In particular $\alpha_{0}=\frac{T_{k+r}}{T_{r}}$, $\alpha_{1}=\frac{T_{2 k+r}}{T_{k+r}}$, and $\beta_{i}=\left\langle b_{k} ; \alpha_{i-1}\right\rangle$ if $i=1,2$.

(3) $\lim _{n \rightarrow \infty} \frac{T_{n+k}}{T_{n}}=\lim _{t \rightarrow \infty} \alpha_{t}[i]=a_{k}+\lim _{t \rightarrow \infty} \frac{N_{t-1}(t-1)}{D_{t-1}(t-1)}$.

For instance, $\frac{T_{80}}{T_{70}}=\frac{T_{10(8)}}{T_{10(7)}}=\alpha_{7}=\alpha_{7}^{[6]}=443+\frac{\hat{N}_{6}(6)}{\hat{D}_{6}(6)}$, where $\hat{N}_{2}(2)=41$, $\hat{D}_{2}(2)=443, \quad \hat{N}_{3}(3)=41+\frac{1}{443}=\frac{18164}{443}, \hat{D}_{3}(3)=443+\frac{41}{443}=\frac{196290}{443}, \hat{N}_{4}(4)=$ $41+\frac{443}{196290}=\frac{8048333}{196290}, \hat{D}_{4}(4)=443+\frac{18164}{196290}=\frac{86974634}{196290}, \hat{N}_{5}(5)=\frac{3566156284}{86974634}$, $\hat{D}_{5}(5)=\frac{38537811195}{86974634}, \hat{N}_{6}(6)=\frac{1580137233629}{38537811195}$, and $\hat{D}_{6}(6)=\frac{17075816515669}{38537811195}$. These fractions converge to 0.09253 , so $\frac{T_{80}}{T_{70}} \approx 443.09253$. It can be compared to the $\operatorname{root} \frac{1}{3}(443+\sqrt[3]{87022054+1914 \sqrt{33}}+\sqrt[3]{87022054-1914 \sqrt{33}}) \approx 443.09253$ of $x^{3}=443 x^{2}+41 x+1$ as well as to $1.839286755^{10} \approx 443.09253$.

\section{Acknowledgements}

This is supported by HanNam University research fund, 2015.

\section{References}

[1] M. Abrate, S. Barbero, U. Cerruti, N. Murru, Periodic representations for cubic irrationalities, Fibo. Quart. 50(3)(2012), 252-264.

[2] E. Choi, J. Jo, Identities involving tribonacci numbers, J. Chungcheng Math. Soc. 28(2015), 39-51.

[3] F.H. Daus, Normal ternary continued fraction expansions for the cube roots of integers. Amer. J. Mathematics, 44(4)(1922), 279-296.

[4] A.K. Gupta, A.K. Mittal, Bifurcating continued fractions,(2000), math GM/000222\%.

[5] A.K. Mittal, A.K. Gupta, Bifurcating continued fractions II,(2000), math GM/0008060. 
[6] S. Finch, Mathematical Constants, Encyclopedia of mathematics and its applications, Cambridge University(2003), 4-8.

[7] C. Hermite, Extraits de lettres de M. Ch. Hermite a M. Jacobi sur differents objets de la theorie des nombres, J. Reine Angew. Math. 40(1850), 286.

[8] I. Stewart, The mathematics of life, Basic Books,(2001), 43-45. 
